\section{Two techniques for guide wire advancement along a tortuous pancreatic duct: the through-the-stent and flipped "U-wire" techniques}
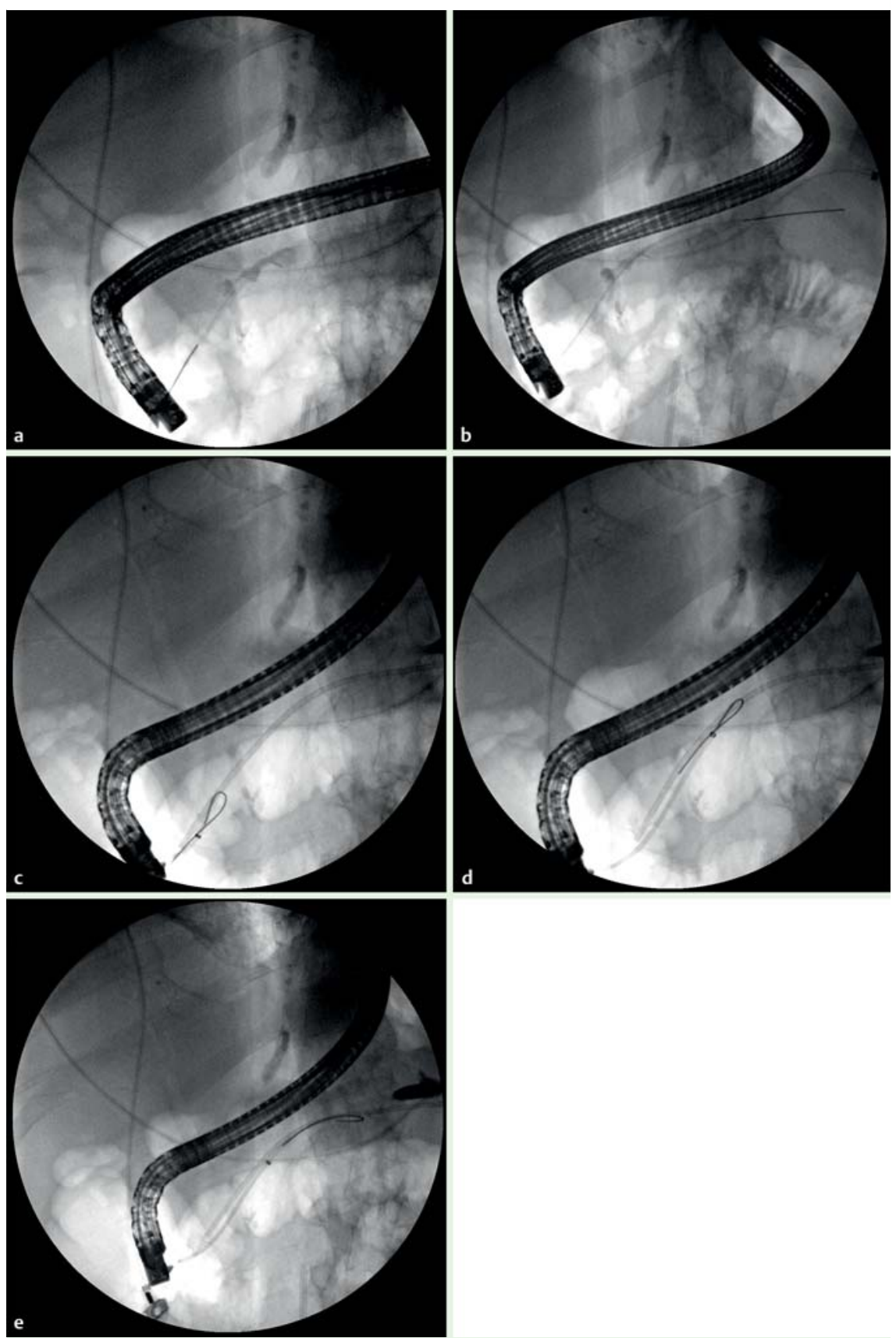

Fig. 1 Images taken during endoscopic retrograde cholangiopancreatography (ERCP) performed in a 52-year-old man with a pseudocyst that had been drained transgastrically showing: $\mathbf{a}$ the wire being advanced through the stent and into the pancreatic duct in the tail of the pancreas; $\mathbf{b}$ the wire in the pancreatic duct after being passed through the stent; $\mathbf{c}$ the wire after its tip had been flipped outside the papilla ready to be introduced into the pancreatic duct; $\mathbf{d}$ the flipped wire being advanced carefully into the pancreatic duct; e the wire reaching the pancreatic duct in the region of the pancreatic tail.
A 52-year-old man with a history of renal transplantation, immunosuppression, and valvular heart disease developed severe acute pancreatitis with a pancreatic pseudocyst. Endoscopic retrograde cholangiopancreatography (ERCP) performed 6 weeks previously had revealed a large pancreatic duct leak requiring the placement of a plastic stent.

A follow-up ERCP and endoscopic ultrasound (EUS) were performed because of the enlarging pseudocyst. The pseudocyst was drained transgastrically using EUS guidance. During ERCP the pancreatic duct was selectively cannulated using a biliary cannula and guide wire (Jagwire). However, it was not possible to pass the guide wire far enough into the pancreatic duct, so instead the guide wire was advanced into the pancreatic tail through the stent ( $\bullet$ Fig. 1 a, b).

The stent was removed over the wire using a snare (snare-over-the-wire technique) and the biliary cannula was then advanced over the wire. Once the cannula was in the pancreatic duct, a complete pancreatogram was performed. This revealed a leak at the level of the body of the pancreas, so larger caliber stents were therefore placed. Initially a 7-Fr $15-\mathrm{cm}$ plastic pancreatic stent was inserted. Repeat cannulation of the pancreatic duct with the wire was possible; however, the wire had a tendency to stick and coil within a tortuously dilated and fibrotic segment of the pancreatic duct at the level of the head. The wire was therefore removed and its floppy tip was flipped at the level of the papilla ( Fig. 1c). The flipped wire was then advanced carefully into the pancreatic duct ( Fig.1d,e). Because of the "Ushape" in the flipped wire, its advancement along the duct went smoothly. No entrapment occurred in the tortuously dilated segment, which allowed the floppy tip to be smoothly advanced towards the pancreatic duct within the tail of the pancreas. Once the wire was within the tail area, its tip was straightened and the biliary catheter was removed. A second plastic stent was placed over the wire into the pancreatic duct to optimize transpapillary drainage of the pseudocyst and to improve the chances of sealing the pancreatic duct leak.

Deep cannulation of the pancreatic duct is essential in obtaining an adequate pancreatogram and for the placement of pancreatic stents. Herein, we have described two useful tips that can be used to advance a wire deep into the pancreatic duct. First, in the presence of an in situ 
stent, the wire can be advanced through the stent. The stent can then be removed over the wire, and a biliary cannula can be advanced over the wire, deep into the pancreatic duct, so facilitating an adequate pancreatogram. The second technique of wire placement involves flipping the tip of the wire to create a "U-shape", which then enables the wire to be passed deep into the pancreatic duct.

Although the "U-wire" technique has been used to cannulate the common bile duct in patients with previous metal stents [1], we are not aware of any reports describing the above techniques for pancreatic duct cannulation. We therefore believe that these two techniques for wire placement should be added to the armamentarium of the therapeutic pancreatic endoscopist.
Endoscopy_UCTN_Code_TTT_1AR_2AI

Competing interests: None

\section{K. Mönkemüller, D. Popa, J. Ramesh, C. M. Wilcox}

Division of Gastroenterology and Hepatology, Basil Hirschowitz Endoscopic Center of Excellence, University of Alabama, Birmingham, Alabama, USA

\section{References}

1 Liu F, Zhu JY, Li ZS. The "U-wire" technique during ERCP for easy guide wire passage through occluded uncovered metal stents. Endoscopy 2012; 44: E116

\section{Bibliography}

DOI http://dx.doi.org/

10.1055/s-0033-1344066

Endoscopy 2013; 45: E183-E184

(c) Georg Thieme Verlag KG

Stuttgart · New York

ISSN 0013-726X

\section{Corresponding author}

\section{K. Mönkemüller, MD, PhD}

Division of Gastroenterology and Hepatology Basil Hirschowitz Endoscopic Center of Excellence Endoscopy Unit, JT 664

619 19th Street South

Birmingham

AL 35249

USA

Fax: +1-205-934-1537

klaus1@uab.edu 